\title{
Abdominal Cocoon With Small Bowel Obstruction: A Rare Case Report
}

\author{
An-Shu $\mathrm{xu}^{1}$, xiao-bin yang$^{1}$, chao-chun $\mathrm{fu}^{1}$, yong hou ${ }^{1}$, and li-hua $\mathrm{zhou}^{1}$ \\ ${ }^{1}$ The First People's Hospital of Qujing City, Yunnan Province
}

May 6, 2020

\begin{abstract}
Abdominal cocoon is a rare disease,that is characterized by a thick,fibrotic and cocoon-like menbrane encapsulating total or partially the bowel.Tts etiology remains unknown.Surgical management is the rteatment of choice.In this report, it is described a patient with abdominal cocoon.
\end{abstract}

\section{Abdominal Cocoon With Small Bowel Obstruction: A Rare Case Report}

An-Shu $\mathrm{Xu}^{1}$, Xiao-Bin Yang ${ }^{2}$, Chao-Chun $\mathrm{Fu}^{2 *}$, Hou Yong ${ }^{1}, \mathrm{Li}_{-} \mathrm{Hua}$ Zhou ${ }^{1}$

Department of Surgery, 2. Department of Gastrointestinal, The First People's Hospital, No. 1 Yuanlin Road, Qujing City, Yunnan Province 655000, China

Corresponding author:Chao-Chun Fu,903130686@qq.com

\section{Key clinical message}

Abdominal cocoon is a rare disease, that is characterized by a thick,fibrotic and cocoon-like menbrane encapsulating total or partially the bowel.Tts etiology remains unknown.Surgical management is the rteatment of choice.

\section{KEYWORDS}

Abdominal Cocoon,Small Bowel Obstruction

\section{CASE PRESENTATION}

Patient: Dong Moumou, gender: male, age: 51 years old, admitted to hospital for "intestinal obstruction" due to "abdominal bloating for more than 40 years, worsening nausea and vomiting and admitted to hospital for 20 days". After repeated treatments in the external hospital, abdominal pain improved, but repeated attacks. Physical examination: Vital signs were stable. Normal development, moderate nutrition, step into the ward, take the lead. Abdominal bulge, abdominal breathing, no intestinal and peristaltic waves, soft abdomen, no tenderness in the abdomen, untouched liver, spleen, and kidneys, Murphy's sign (-), no percussion in the liver area and both kidney areas. Mobile dullness (-), bowel sounds 3 times / min, no abdominal murmur. Auxiliary examination: CT prompts: manifested as small intestine clustering, dilation of lumen, and fluid accumulation. Cocoon-like, ring-shaped low-density fiber envelopes can be seen around the lesions. After enhancement, the envelopes are strengthened. Initial diagnosis: intestinal obstruction, abdominal cocoon disease is possible. Figure 1. Enteroscopy tips: narrowing of the small intestine at the end of the ileum. What was seen during the operation: During the operation, the omentum was missing. During the operation, the small ileum and sigmoid colon of the ileum were surrounded by a gray, smooth, tough and thick fibrous membrane, which looked like a silkworm cocoon. There is also fibrous membrane adhesion 
between the intestine and the intestine. The small intestine could not be separated. The small intestine of the ileum was resected and intestinal anastomosis was performed. The sigmoid colon was subjected to fibrous membrane resection and intestinal adhesions were loosened. Intraoperative as shown in Figure 2. After the operation, the specimen was broken open and the small intestine enveloped as shown in Figure 3. Postoperative medical examination supports intraoperative diagnosis. The patient recovered well and was discharged smoothly.

\section{DISCUSSION}

Abdominal cocoon disease is a rare abdominal disease characterized by part or all of the organs in the abdominal cavity surrounded by a layer of membrane-like material, shaped like a silkworm cocoon, first reported and named by Foo et al. [1] in 1978. The author admitted this patient because of congenital factors. The patient has had repeated abdominal pain since childhood. It is believed that this patient is a congenital peritoneal malformation, which is caused by the peritoneal variability during embryonic development, and the patient complicated with omentum absent, the sigmoid colon is also wrapped. Most scholars believe that pre-operative diagnosis is difficult, but abdominal CT diagnosis is of great help. The author may diagnose pre-operative CT diagnosis of abdominal cocoon disease. Intraoperative and postoperative medical examinations have further confirmed this disease. The treatment includes conservative medical treatment and surgical treatment. The author of this patient used surgical treatment. During the operation, the remaining small intestine after the removal of the diseased small intestine did not cause short bowel syndrome, so the diseased small intestine was directly removed. The adhesion of the sigmoid colon is not heavy, so only adhesion release is performed.

\section{CONFLICTS OF INTEREST}

The authors declare no conflicts of interest.

\section{Informed Consent}

Informed consent has been obtained for the publication of this clinical image.

\section{AUTHOR CONTRIBUTIONS}

ASX, XBY, and CCF: treated the patient. YH and LHZ performed the histological analysis. ASX, HZL, and YH: wrote the paper.

\section{References}

1.Foo $\mathrm{KT}, \mathrm{Ng} \mathrm{KC,Rauff} \mathrm{A,et} \mathrm{al.Unusual} \mathrm{small} \mathrm{intestinal} \mathrm{obstruction} \mathrm{in} \mathrm{adolescent} \mathrm{girls:} \mathrm{the} \mathrm{abdominal} \mathrm{co-}$ coon.Br J Surg,1978,65:427-430.

Fig 1 CT performance 


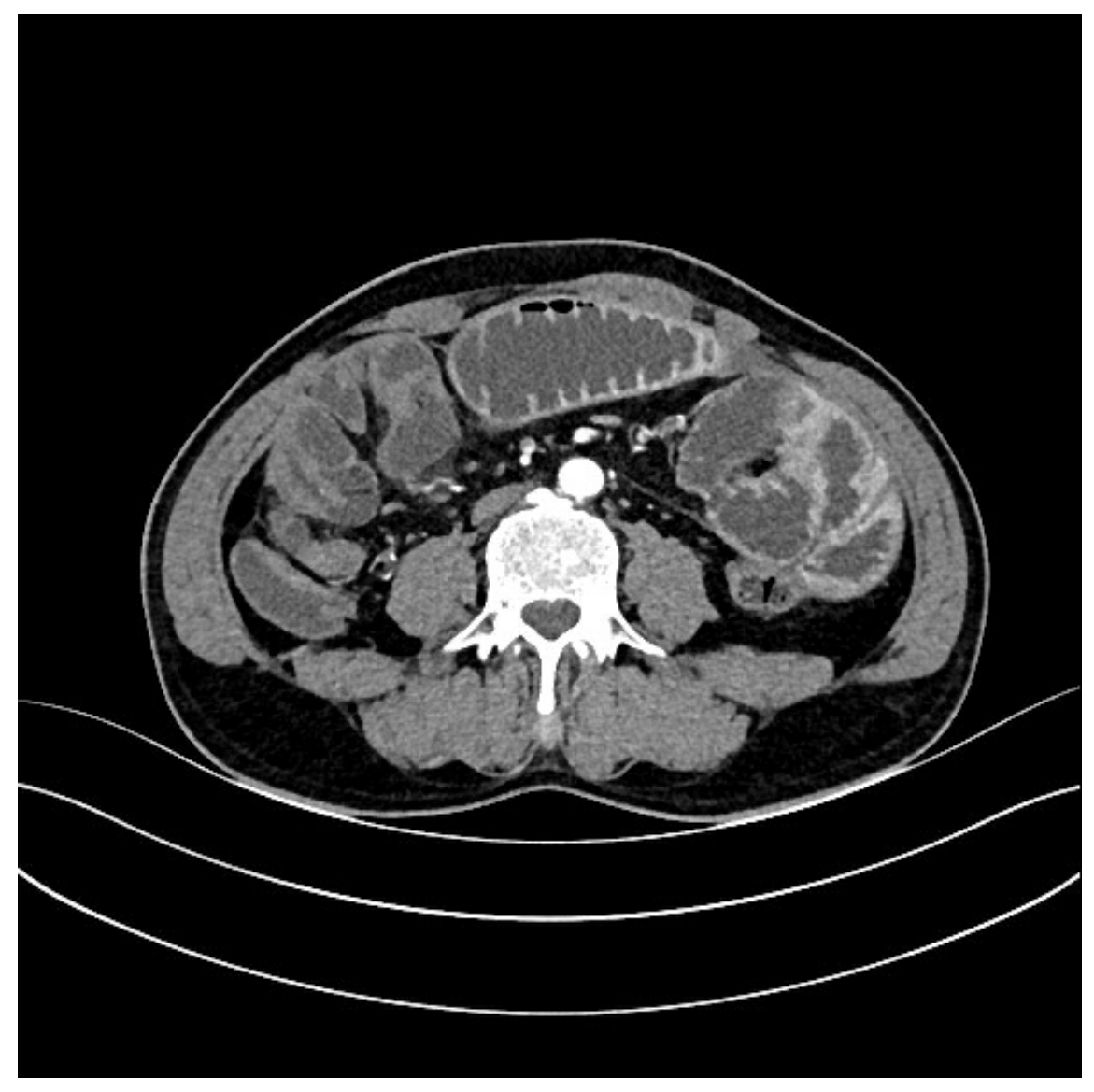

In the picture, see small bowel obstruction, thickening of the small bowel wall.

Fig 2 What I saw during the operation 


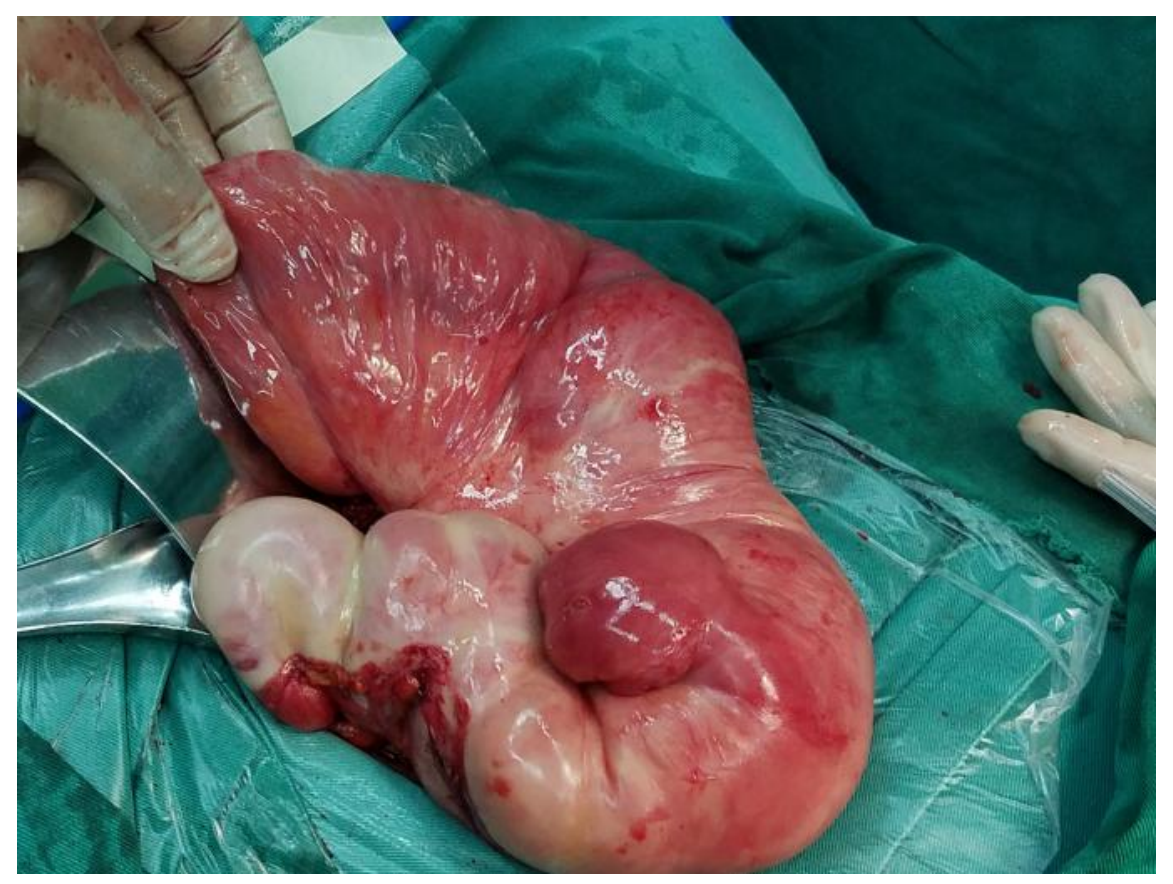

the small ileum of the ileum were surrounded by a gray, smooth, tough and thick fibrous membrane, which looked like a silkworm cocoon.

Fig 3 Seen through the specimen

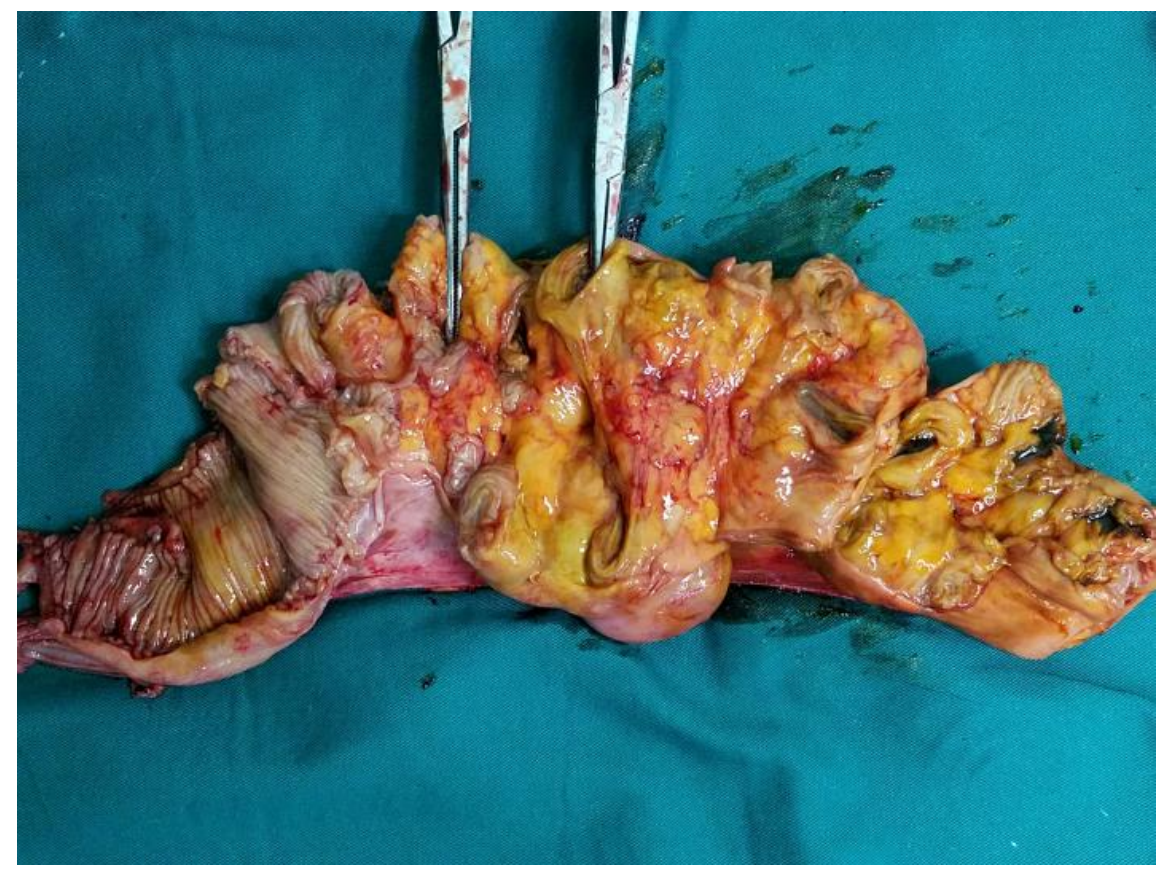

The small intestine is wrapped and curved, forming an intestinal obstruction. 